\title{
THE EXISTENCE OF MINIMAL REGULAR LOCAL OVERRINGS FOR AN ARBITRARY DOMAIN
}

\author{
BERNARD JOHNSTON
}

\begin{abstract}
It is shown that the set of regular local rings of dimension $n$ containing an integral domain $D$, having the same quotient field as $D$, and ordered by containment satisfies the descending chain condition. The set of regular local rings of dimension $n$ contained in a regular local ring of dimension $m, n>m$, need not satisfy the ascending chain condition, as is shown by example.
\end{abstract}

Introduction. The local factorization theorem of Zariski and Abhyankar states that if $R$ and $S$ are 2-dimensional regular local rings ("RLR's") with $R \subseteq S \subset$ quotient field of $R$, then $S$ is obtainable from $R$ by a unique finite sequence of quadratic transforms [7, Lemma, p. 538; 1, Theorem 3]. This result does not extend to higher dimensions, as was shown by Sally [5, Corollary 4.5] and Shannon [6, Example 3.2]. Nevertheless one can conclude that for any such $R$ and $S$ as above with $\operatorname{dim} R=\operatorname{dim} S=n \geq 2$, every chain of RLR's between $R$ and $S$ is finite [2, Corollary 4.10]. The proof of this result involves showing: (a) the ascending chain condition ("a.c.c."), and (b) the descending chain condition ("d.c.c.") hold for the set of RLR's between $R$ and $S$, ordered by containment. (Note that such rings are necessarily of dimension $n$ [5, Lemma 5.3].) Such behavior does not occur for more general classes of local domains [2, Examples 4.9 and 5.2].

If $R$ and $S$ have different dimensions, say $\operatorname{dim} R=m>n=\operatorname{dim} S$, then the a.c.c. no longer holds on the set of $m$-dimensional RLR's between $R$ and $S$, as is shown in Example 2. The main result of this paper is that the d.c.c. continues to hold on the set of $n$-dimensional RLR's between $R$ and $S$ and, in fact, given any integral domain $D$, if we denote by $\mathfrak{R}$ the set of RLR's of dimension $n$ which properly contain $D$ and are contained in the quotient field of $D$, then $\Re$ satisfies the d.c.c. In particular, this shows that if $S \in \mathfrak{R}$ then there exists $S^{\prime} \in \mathfrak{R}$ such that $S \supseteq S^{\prime} \supset R$ and such that there is no RLR $T$ with $S^{\prime} \supset T \supset R$. Examples of such "minimal" RLR's are given in [5, Theorem 5.1, 2, Example 5.4].

NotATION. We assume throughout that $\left(S_{0}, \mathcal{N}_{0}, k_{0}\right) \supset\left(S_{1}, \mathcal{N}_{1}, k_{1}\right) \supset \cdots$ is a strictly descending sequence of quasilocal (commutative) rings (i.e., $\mathcal{N}_{i}$ is the unique maximal ideal of $S_{i}$ ) such that $\mathcal{N}_{i} \cap S_{i+1}=\mathcal{N}_{i+1}$ for all $i$. Then the residue field $k_{i}$ canonically embeds in $k_{j}$, for $i \geq j$, and we identify $k_{i}$ with its image in order to assume $k_{0} \supseteq k_{1} \supseteq \cdots$. We denote $\bigcap_{i=0}^{\infty} S_{i}$ by $S_{\infty}$ and $\bigcap_{i=0}^{\infty} \mathcal{N}_{i}$ by $\mathcal{N}_{\infty}$.

We define the $i$ th order function for $x \in S_{i}$ by $\operatorname{ord}_{i}(x):=\sup \left\{r \in \mathrm{N} \mid x \in \mathcal{N}_{i}^{r}\right\}$. By the embedding dimension of $S_{i}$ ("emdim $S_{i}$ ") we indicate the dimension of

Received by the editors May 5, 1986.

1980 Mathematics Subject Classification (1985 Revision). Primary 13H05; Secondary 13E99, $14 \mathrm{E} 40$.

Key words and phrases. Birational, regular local ring, quadratic transform, descending chain condition. 
$\mathcal{N}_{i} / \mathcal{N}_{i}^{2}$ as a $k_{i}$-vector space. By a regular local ring we mean a Noetherian quasilocal domain $R$ such that $\operatorname{dim} R=\operatorname{emdim} R$. If $i \geq j$, we define the $k_{j}$-vector space $V_{j, i}$ to be

$$
\left(\mathcal{N}_{i} S_{j}+\mathcal{N}_{j}^{2}\right) / \mathcal{N}_{j}^{2} \subseteq \mathcal{N}_{j} / \mathcal{N}_{j}^{2}=: V_{j, j}
$$

We denote the extended Rees ring of $S_{i}$ with respect to $\mathcal{N}_{i}$, namely the ring $S_{i}\left[\mathcal{N}_{i} t, t^{-1}\right]$, where $t$ is an indeterminate, by $R\left(S_{i}\right)$. If $i \geq j$ then $R\left(S_{i}\right) \subseteq R\left(S_{j}\right)$. We denote the associated graded ring of $S_{i}$ with respect to $\mathcal{N}_{i}$, namely the ring $\bigoplus_{r=0}^{\infty} \mathcal{N}_{i}^{r} / \mathcal{N}_{i}^{r+1}$, by $S_{i}^{*}$. Then $S_{i}^{*}=R\left(S_{i}\right) /\left(t^{-1}\right) \mathcal{R}\left(S_{i}\right)$. If $i \geq j$ we have a canonical ring homomorphism $\varphi_{i j}: S_{i}^{*} \rightarrow S_{j}^{*}$ given by

$$
\begin{aligned}
R\left(S_{i}\right) /\left(t^{-1}\right) R\left(S_{i}\right) & \rightarrow R\left(S_{i}\right) /\left(t^{-1}\right) R\left(S_{j}\right) \cap R\left(S_{i}\right) \\
& \hookrightarrow R\left(S_{j}\right) /\left(t^{-1}\right) R\left(S_{j}\right)=S_{j}^{*} .
\end{aligned}
$$

We proceed to the proof of the main result. We first consider four simple lemmas.

LEMMA 1. With the notation as above, assume in addition that emdim $S_{i}=$ $n_{i}<\infty$ for all $i$. Then there exists a cofinal subset $C$ of the nonnegative integers such that, for $i, j, k \in C$ with $i>j>k$, we have $V_{k, j}=V_{k, i}$.

PrOOF. We have $V_{0,0} \supseteq V_{0,1} \supseteq \cdots$. Since $\operatorname{dim}_{k_{0}} V_{0,0}=n_{0}<\infty$, this is a descending sequence of finite $k_{0}$-vector spaces. Thus there exists $r_{0}>0$ such that $V_{0, r_{0}}=V_{0, r_{0}+1}=\cdots$.

Now consider the descending sequence of $k_{r_{0}}$-vector spaces: $V_{r_{0}, r_{0}} \supseteq V_{r_{0}, r_{0}+1} \supseteq$ $\cdots$. Then there exists $r_{1}>r_{0}$ such that $V_{r_{0}, r_{1}}=V_{r_{0}, r_{1}+1}=\cdots$. We continue this process recursively, and let $C:=\left\{0, r_{0}, r_{1}, \ldots\right\}$.

By renumbering we may and do assume henceforth that the set $C$ in Lemma 1 is actually equal to $\mathbf{N}$. For $i>j$ we denote the $k_{j}$-vector space $V_{j, i}$ by $V_{j}\left(\subseteq V_{j, j}\right)$.

LEMMA 2. With the notation and assumptions as above, for $i>j$ there exists a surjective $k_{i}$-vector space homomorphism $\psi_{i j}: V_{i} \otimes_{k_{i}} k_{j} \rightarrow V_{j}$.

In particular, setting $d_{i}:=\operatorname{dim}_{k_{i}} V_{i}$, we have $0 \leq d_{0} \leq d_{1} \leq \cdots$.

ProOF. Let $k>i$. We denote elements of $V_{j}=\left(\mathcal{N}_{k} S_{j}+\mathcal{N}_{j}^{2}\right) / \mathcal{N}_{j}^{2}$ by $\bar{a}$, where $a$ may be chosen to lie in $\mathcal{N}_{k} S_{j}$. We define $\psi_{i j}$ by $\bar{a} \otimes \alpha \mapsto \alpha \bar{a}$. Here, the first bar denotes the image of $a$ (modulo $\mathcal{N}_{i}^{2}$ ) and the second bar denotes the image of $a$ (modulo $\mathcal{N}_{j}^{2}$ ). Since $\mathcal{N}_{i}^{2} \subseteq \mathcal{N}_{j}^{2}$ and since this mapping is bilinear, $\psi_{i j}$ is well defined.

If $\bar{a} \in V_{j}$ as above, we have $a=\sum a_{h} s_{h}$, where $a_{h} \in \mathcal{N}_{k}, s_{h} \in S_{j}$. We may assume that $s_{h} \notin \mathcal{N}_{j}$. Then setting $\alpha_{h}:=\bar{s}_{h}$ (modulo $\left.\mathcal{N}_{j}\right) \in k_{j}$, we have $\psi_{i j}\left(\sum \bar{a}_{h} \otimes \alpha_{h}\right)=$ $\bar{a}$ (where, again, the first bar indicates "modulo $\mathcal{N}_{i}^{2}$ " and the second "modulo $\left.\mathcal{N}_{j}^{2} "\right)$.

DEFinition. Let $L_{i}: S_{i} \rightarrow S_{i}^{*}$ be the function defined by

$$
L_{i}(a)= \begin{cases}\bar{a} \in \mathcal{N}_{i}^{r} / \mathcal{N}_{i}^{r+1}, & \text { if } \operatorname{ord}_{i}(a)=r<\infty, \\ 0, & \text { otherwise. }\end{cases}
$$

NOTE. If $\operatorname{ord}_{i}(a)=\operatorname{ord}_{i}(b)$ and $L_{i}(a)+L_{i}(b) \neq 0$, then $L_{i}(a+b)=L_{i}(a)+L_{i}(b)$, and if $L_{i}(a) L_{i}(b) \neq 0$, then $L_{i}(a b)=L_{i}(a) L_{i}(b)$. If $\operatorname{ord}_{i}(a)=r<\infty, i \geq j$, then

$$
\varphi_{i j}\left(L_{i}(a)\right)= \begin{cases}L_{j}(a), & \text { if } \operatorname{ord}_{j}(a)=r_{j}, \\ 0, & \text { otherwise. }\end{cases}
$$

(Since $\mathcal{N}_{j}^{r} \supseteq \mathcal{N}_{i}^{r}, \operatorname{ord}_{j}(a) \geq \operatorname{ord}_{i}(a)$ whenever $i \geq j$.) 
Notation. If emdim $S_{i}=n_{i}<\infty$ then we may choose $x_{i 1}, \ldots, x_{i n_{i}} \in \mathcal{N}_{i}$ such that the images of $x_{i 1}, \ldots, x_{i d_{i}}$ (modulo $\mathcal{N}_{i}^{2}$ ) form a basis for $V_{i}$. We have $S_{i}^{*}=$ $k_{i}\left[L_{i}\left(x_{i 1}\right), \ldots, L_{i}\left(x_{i n_{i}}\right)\right]$, and we denote by $S_{i}^{\prime}$ the subring $k_{i}\left[L_{i}\left(x_{i 1}\right), \ldots, L_{i}\left(x_{i d_{i}}\right)\right]=$ $k_{i}\left[V_{i}\right]$.

LEMMA 3. With the notation and assumptions as above, for $i \geq j$ there exists a $k_{i}$-algebra surjection $\theta_{i j}: S_{i}^{\prime} \otimes_{k_{i}} k_{j} \rightarrow S_{j}^{\prime}$.

ProOF. It suffices by linearity to define $\theta_{i j}$ for homogeneous elements of $S_{i}^{\prime}$. If $L_{i}(a) \in S_{1}^{\prime}$ is one such and $\alpha \in k_{j}$, we define $\theta_{i j}\left(L_{i}(a) \otimes \alpha\right):=\alpha \varphi_{i j}\left(L_{i}(a)\right)$. Since $\varphi_{i j}\left(V_{i}\right)=\psi_{i j}\left(V_{i}\right) \subseteq V_{j}$ (as $k_{i}$-vector space homomorphisms), it is routine to check that $\theta_{i j}$ is well defined.

Surjectivity of $\theta_{i j}$ follows from surjectivity of $\psi_{i j}$ and the following commutative diagram:

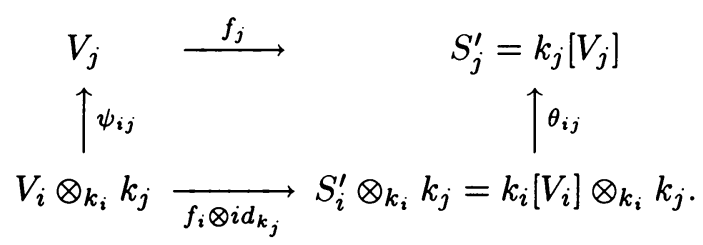

LEMMA 4. With the notation and assumptions as in Lemma 3, assume in addition that $\theta_{i j}$ is an isomorphism for all $i, j$. Then $a \in \mathcal{N}_{\infty}$ implies $L_{0}(a) \in S_{0}^{\prime}$.

PROOF. If $\operatorname{ord}_{0}(a)=\infty$ this is clear, so we may assume without loss of generality that $\operatorname{ord}_{0}(a)<\infty$. Since $\operatorname{ord}_{0}(a) \geq \operatorname{ord}_{1}(a) \geq \cdots>0$, there exists $N \gg 0$ such that $\operatorname{ord}_{N}(z)=\operatorname{ord}_{N+1}(a)=\cdots=: m$. If $l>N$ we get $\varphi_{l N}\left(L_{l}(a)\right)=L_{N}(a) \in S_{N}^{\prime} \backslash\{0\}$. Since $S_{N}^{\prime}$ injects into $S_{N}^{\prime} \otimes k_{N} k_{0}$ and since, by hypothesis, $\theta_{N 0}: S_{N}^{\prime} \otimes_{k_{N}} k_{0} \stackrel{\sim}{\rightarrow} S_{0}^{\prime}$, we have that

$$
\theta_{N 0}\left(L_{N}(a) \otimes 1\right)=1 \cdot \varphi_{N 0}\left(L_{N}(a)\right) \neq 0
$$

so

$$
\varphi_{N 0}\left(L_{N}(a)\right)=L_{0}(a) \in S_{0}^{\prime}
$$

THEOREM. If $\left(S_{0}, \mathcal{N}_{0}, k_{0}\right) \supset\left(S_{1}, \mathcal{N}_{1}, k_{1}\right) \supset \cdots$ is a strictly descending sequence of RLR's, all of which have the same dimension $n$ and the same quotient field $K$, then the quotient field of the intersection $S_{\infty}$ is properly contained in $K$.

ProOF. Applying Lemma 1 to this sequence of rings and omitting all rings whose indices are not in the set $C$ defined in Lemma 1 (which does not change $S_{\infty}$ ), we may assume that $V_{j, i}=V_{j, l}=: V_{i}$ for $i, l>j$. By Lemma 2, we have that $0 \leq d_{0} \leq d_{1} \leq \cdots \leq n$, since $V_{i} \subseteq V_{i, i}$ for all $i$, and since $\operatorname{dim}_{k_{i}} V_{i, i}=n$ by regularity. Thus there exists $N \in \mathbf{N}$ such that $d_{N}=d_{N+1}=\cdots=: d$. By renumbering once more, we assume $N=0$.

Note that $d<n$, for $d=n$ would yield, e.g., that $\operatorname{dim}_{k_{0}}\left(\mathcal{N}_{1} S_{0}+\mathcal{N}_{0}^{2}\right) / \mathcal{N}_{0}^{2}=n$, but then Nakayama's Lemma gives $\mathcal{N}_{1} S_{0}=\mathcal{N}_{0}$ and Zariski's Main Theorem [4, (37.4)] forces $S_{1}=S_{0}$, a contradiction.

Now, for all $i$, let $\left\{x_{i 1}, \ldots, x_{i n}\right\}$ be a minimal generating set for $\mathcal{N}_{i}$ such that the images of $x_{i 1}, \ldots, x_{i d}$ in $V_{i, i}$ form a basis for $V_{i}$. Then $S_{i}^{*}$ is a polynomial ring over $k_{i}$ of dimension $n$ [8, Theorem 25, p. 301] and $S_{i}^{\prime}$ is a subring of $S_{i}^{*}$ which is itself a 
polynomial ring over $k_{i}$ of dimension $d$, with generators a subset of the generators for $S_{i}^{*}$. Then $S_{i}^{\prime} \otimes_{k_{i}} k_{j}$ is a polynomial ring of dimension $d$ over $k_{j}$, and it follows that the ring homomorphism of Lemma 3 must be an isomorphism. Hence a simple application of Lemma 4 says that $L_{0}(a) \in S_{0}^{\prime}$ whenever $a \in \mathcal{N}_{\infty}$.

Suppose now that $y \in \mathcal{N}_{0}$ such that $x_{i 1}, \ldots, x_{i d}$ and $y$ are linearly independent (modulo $\mathcal{N}_{0}^{2}$ ) over $k_{0}$. Then $y \notin$ q.f. $\left(S_{\infty}\right)$. For, supposing it were, we would have $y=a / b, a, b \in \mathcal{N}_{\infty}$, and so $b y=a$ (in $S_{0}$ ) which gives $L_{0}(b) L_{0}(y)=L_{0}(a)$. But this contradicts that $L_{0}(a), L_{0}(b) \in S_{0}^{\prime}$, since $L_{0}(y) \notin S_{0}^{\prime}$ and since $S_{0}^{\prime} \subset S_{0}^{*}$ are polynomial rings.

EXAMPLE 1. Infinite descending sequences of birationally dominating RLR's do exist, and a typical example is

$$
k[X, Y]_{(X, Y)} \supset k[X, X Y]_{(X, X Y)} \supset \cdots \supset k\left[X, X^{i} Y\right]_{\left(X, X^{i} Y\right)} \supset \cdots,
$$

where $k$ is a field and $X$ and $Y$ are indeterminates.

COROLLARY. If $D$ is an integral domain then the set of $R L R$ 's of dimension $n$ which contain $D$ birationally satisfies the d.c.c. if ordered by containment.

PROOF. If not, there would exist an infinite strictly descending sequence of RLR's containing $D$ birationally. By the theorem, the intersection of this sequence would be a domain having a strictly smaller quotient field, which contradicts the birationality.

EXAMPLE 2. The "dual" of the theorem does not hold, i.e., given an $m$ dimensional RLR $R$ containing an $n$-dimensional RLR $S$ birationally, then if $m<n$ there may exist an infinite strictly ascending chain of RLR's of dimension $n$ between $R$ and $S$. Let $k$ be a field and set $D:=k[[X]]$, where $X$ is an indeterminate. Pick $Y \in D$ such that $X$ and $Y$ are algebraically independent over $k$ and let $V:=D \cap k(X, Y)$. Then $V$ is a rank one discrete valuation ring (i.e., a 1-dimensional RLR) which dominates the 2-dimensional $\mathrm{RLR} R:=k[X, Y]_{(X, Y)}$ birationally. If we let $R_{i}$ denote the $i$ th quadratic transform of $R$ along $V$, then the sequence $R=R_{0} \subset R_{1} \subset \cdots$ is finite if and only if there exists an $i$ such that ht $\left(\mathcal{M}_{v} \cap R_{i}\right)=1$, where $\mathcal{M}_{v}$ denotes the unique maximal ideal of $V$. In this case we have $V=\left(R_{i}\right)_{\mathcal{M}_{v} \cap R_{i}}$, and $V$ is a spot over $R$. But this contradicts the dimension formula [3, Theorem 23] since $\operatorname{dim} V+\operatorname{trdeg}_{R / \mathcal{M}_{R}} V / \mathcal{M}_{V}=1+0<2=\operatorname{dim} R$, and the universally catenarian property of $R$ implies an equality here. By adjoining variables, this example extends to higher dimensions.

In this connection it is interesting to speculate on what the structure of the ring $S_{\infty}$ might be where $S_{0} \supset S_{1} \supset \cdots$ is an arbitrary descending chain of birational RLR's. For example, need it be Noetherian? The "dual" question concerns the nature of the union of an ascending chain of RLR's contained in the same quotient field. Some material along these lines can be found in $[6, \S 4]$.

\section{REFERENCES}

1. S. Abhyankar, On the valuations centered in a local domain, Amer. J. Math. 78 (1956), 321-348.

2. B. Johnston, A finiteness condition on regular local overrings of a local domain, Trans. Amer. Math. Soc. 299 (1987), 513-524.

3. H. Matsumura, Commutative algebra (2nd ed.), Benjamin/Cummings, Reading, Mass., 1980.

4. M. Nagata, Local rings, Interscience, New York, 1962.

5. J. Sally, Regular overrings of regular local rings, Trans. Amer. Math. Soc. 171 (1972), 291-300. 
6. D. Shannon, Monoidal transforms of regular local rings, Amer. J. Math. 95 (1973), 294-320.

7. O. Zariski, Reductions of the singularities of algebraic three-dimensional varieties, Ann. of Math. (2) 45 (1944), 472-542.

8. O. Zariski and P. Samuel, Commutative algebra, Vol. II, Van Nostrand, Princeton, N. J., 1960.

Department of Mathematics, Purdue University, West Lafayette, Indiana 47907

Current address: Department of Mathematics, University of Utah, Salt Lake City, Utah 84112 